\title{
INTEGRAL GEOMETRY OF REAL SURFACES IN COMPLEX PROJECTIVE SPACES
}

Dedicated to Professor Katsuhiro Shiohama on his sixtieth birthday

By

Hong Jae KANG and Hiroyuki TASAKI

\section{Introduction}

The purpose of this paper is to give a Poincaré formula of real surfaces in complex projective spaces stated in the following theorem.

THEOREM 1.1. Let $C P^{n}$ be a complex projective space of complex dimension $n, M$ a real submanifold of $C P^{n}$ of real dimension 2 and $N$ a complex submanifold of complex dimension $n-1$. Then we have

$$
\begin{aligned}
\int_{U(n+1)} & \sharp(M \cap g N) d \mu_{U(n+1)}(g) \\
= & \frac{\operatorname{vol}(U(n+1)) \operatorname{vol}(N)}{2 \operatorname{vol}\left(C P^{1}\right) \operatorname{vol}\left(C P^{n-1}\right)} \int_{M}\left(1+\cos ^{2} \theta_{x}\right) d \mu_{M}(x),
\end{aligned}
$$

where $\theta_{x}$ is the Kähler angle of $M$ at $x$. Moreover the above formula holds for $a$ real submanifold $M$ of real dimension $2 n-2$ and a complex submanifold $N$ of complex dimension 1 , where $\theta_{x}$ is the Kähler angle of $T_{x}^{\perp} M$.

One of the oldest results in integral geometry is the Poincare formula for the average of the intersection number of two curves. Many differential geometers have studied the Poincaré formula from various points of view. In particular, $\mathbf{R}$. Howard [1] has generalized this formula in Riemannian homogeneous spaces and obtained the following formula.

THEOREM 1.2. [1] Let $G / K$ be a Riemannian homogeneous space with a $G$-invariant Riemannian metric and take submanifolds $M$ and $N$ of $G / K$. Assume that $\operatorname{dim} M+\operatorname{dim} N=\operatorname{dim}(G / K)$ and that $G$ is unimodular. Then

Received December 9, 1999.

Revised April 28, 2000. 


$$
\int_{G} \sharp(M \cap g N) d \mu_{G}(g)=\iint_{M \times N} \sigma_{K}\left(T_{x}^{\perp} M, T_{y}^{\perp} N\right) d \mu_{M \times N}(x, y),
$$

where $\sigma_{K}\left(T_{x}^{\perp} M, T_{y}^{\perp} N\right)$ is defined by (2.1) below. Furthermore, if $G$ is transitive on the sets of tangent spaces to each of $M$ and $N$ then

$$
\int_{G} \sharp(M \cap g N) d \mu_{G}(g)=\sigma_{K}\left(T_{x_{0}}^{\perp} M, T_{y_{0}}^{\perp} N\right) \operatorname{vol}(M) \operatorname{vol}(N),
$$

where $x_{0}$ and $y_{0}$ are any points of $M$ and $N$, respectively.

Let $C P^{n}$ be a complex projective space of complex dimension $n$. The unitary group $U(n+1)$ acts transitively on $C P^{n}$. The isotropy subgroup of $U(n+1)$ at a point in $C P^{n}$ is $U(1) \times U(n)$. Thus $C P^{n}$ can be realized as a homogeneous space $C P^{n}=U(n+1) /(U(1) \times U(n))$. Let $\mathfrak{u}(n+1)$ be the Lie algebra of $U(n+1)$. Define an inner product on $\mathfrak{u}(n+1)$ by

$$
(X, Y)=-\frac{1}{2} \operatorname{Trace}(X Y) \quad(X, Y \in \mathfrak{u}(n+1)) .
$$

We extend this inner product $(\cdot, \cdot)$ on $\mathfrak{u}(n+1)$ to the left invariant Riemannian metric on $U(n+1)$. Then we obtain a biinvariant Riemannian metric on $U(n+1)$. This biinvariant Riemannian metric on $U(n+1)$ induces a $U(n+1)$ invariant Riemannian metric on $U(n+1) /(U(1) \times U(n))$. R. Howard [1] has also obtained the following formula in the case of $C P^{n}$.

THEOREM 1.3. [1] Let $\boldsymbol{C P}^{n}$ be a complex projective space.

(1) Let $M$ and $N$ be complex submanifolds of $C P^{n}$ of complex dimension 1 and $n-1$. Then

$$
\int_{U(n+1)} \sharp(M \cap g N) d \mu_{U(n+1)}(g)=\frac{\operatorname{vol}(U(n+1)) \operatorname{vol}(M) \operatorname{vol}(N)}{\operatorname{vol}\left(C P^{1}\right) \operatorname{vol}\left(C P^{n-1}\right)} .
$$

(2) Let $M$ be a totally real submanifold of $C P^{n}$ of real dimension 2 and $N a$ complex submanifold of complex dimension $n-1$. Then

$$
\int_{U(n+1)} \sharp(M \cap g N) d \mu_{U(n+1)}(g)=\frac{\operatorname{vol}(U(n+1)) \operatorname{vol}(M) \operatorname{vol}(N)}{\operatorname{vol}\left(\boldsymbol{R} P^{2}\right) \operatorname{vol}\left(\boldsymbol{C} P^{n-1}\right)},
$$

where $\boldsymbol{R}^{2}$ is a 2-dimensional real projective space.

Such complex and totally real submanifolds have constant Kähler angles. In the case (1) the Kähler angle of $M$ is 0 and in the case (2) that of $M$ is $\pi / 2$ and 
$\operatorname{vol}\left(\boldsymbol{R} P^{2}\right)=2 \pi=2 \operatorname{vol}\left(\boldsymbol{C} P^{1}\right)$. Thus the formulas in Theorem 1.3 are special cases of the formula in Theorem 1.1.

On the other hand, the second auther proved the following inequality in [2].

$$
\int_{U(n+1)} \sharp\left(M \cap g C P^{n-1}\right) d \mu_{U(n+1)}(g) \leq \frac{\operatorname{vol}(U(n+1))}{\operatorname{vol}\left(C P^{1}\right)} \operatorname{vol}(M),
$$

where $M$ is a submanifold of $C P^{n}$ of real dimension 2. This follows from Theorem 1.1. In this formula, if $M$ is a complex submanifold of $C P^{n}$ of complex dimension 1 then the equality holds and this is the case of Kähler angle 0 in Theorem 1.1.

\section{Acknowledgements}

We would like to thank Professor Katsuei Kenmotsu for his considerable comments at an early version of this paper. The authers are indebted to the referee for useful comments which led to improvement of this paper.

\section{Preliminaries}

In this section we shall review the Poincaré formula on Riemannian homogeneous spaces given by R. Howard [1].

Let $E$ be a finite dimensional real vector space with an inner product. For two vector subspaces $V$ and $W$ of dimension $p$ and $q$ in $E$, take orthonormal bases $v_{1}, \ldots, v_{p}$ and $w_{1}, \ldots, w_{q}$ of $V$ and $W$, respectively, and define

$$
\sigma(V, W)=\left|v_{1} \wedge \cdots \wedge v_{p} \wedge w_{1} \wedge \cdots \wedge w_{q}\right|
$$

This definition is independent of the choice of orthonormal bases. Furthermore, if $p+q=\operatorname{dim} E$ then

$$
\sigma(V, W)=\sigma\left(V^{\perp}, W^{\perp}\right)
$$

Let $G$ be a Lie group and $K$ a closed subgroup of $G$. We assume that $G$ has a left invariant Riemannian metric that is also invariant under the right actions of elements of $K$. This metric induces a $G$-invariant Riemannian metric on $G / K$. We denote by $o$ the origin of $G / K$. For $x$ and $y$ in $G / K$ and vector subspaces $V$ and $W$ in $T_{x}(G / K)$ and $T_{y}(G / K)$, we define $\sigma_{K}(V, W)$ by

$$
\sigma_{K}(V, W)=\int_{K} \sigma\left(\left(d g_{x}\right)_{o}^{-1} V, d k_{o}^{-1}\left(d g_{y}\right)_{o}^{-1} W\right) d \mu_{K}(k)
$$


where $g_{x}$ and $g_{y}$ are elements of $G$ such that $g_{x} o=x$ and $g_{y} o=y$. This definition is independent of the choice of $g_{x}$ and $g_{y}$ in $G$ such that $g_{x} o=x$ and $g_{y} o=y$.

Proposition 2.1. Let $V$ and $W$ be vector spaces with inner product. Assume $\operatorname{dim} V=m \geq n=\operatorname{dim} W$. Let $F: V \rightarrow W$ be a linear mapping. We set $J F=$ $\sup \left\{\left|F\left(u_{1}\right) \wedge \cdots \wedge F\left(u_{n}\right)\right| \mid u_{1}, \ldots, u_{n}\right.$ is orthonormal system of $\left.V\right\}$. If $F$ is not onto mapping then $J F=0$. And if $F$ is onto then for any basis $v_{1}, \ldots, v_{n}$ of $(\operatorname{Ker} F)^{\perp}$,

$$
J F=\frac{\left|F\left(v_{1}\right) \wedge \cdots \wedge F\left(v_{n}\right)\right|}{\left|v_{1} \wedge \cdots \wedge v_{n}\right|}
$$

We can easily show this proposition and omit its proof. We will use this later in calculating the coarea formula.

Let $\boldsymbol{C}^{n}$ be an $n$-dimensional complex vector space with standard real inner product $\langle\cdot, \cdot\rangle$ and almost complex structure $J$. Let $V$ be a vector subspace of $C^{n}$ of real dimension 2. If $v_{1}, v_{2}$ is an orthonormal basis of $V$ then $-1 \leq$ $\left\langle J v_{1}, v_{2}\right\rangle \leq 1$. In the case of $-1 \leq\left\langle J v_{1}, v_{2}\right\rangle<0$, we choose $-v_{2}$ instead of $v_{2}$. Then $0 \leq\left\langle J v_{1}, v_{2}\right\rangle \leq 1$. We set

$$
\theta=\cos ^{-1}\left\langle J_{1}, v_{2}\right\rangle .
$$

We call $\theta$ the Kähler angle of $V$. We remark that the definition of $\theta$ is independent of the choice of the orthonormal basis of $V$. In particular, the vector subspace $V$ is complex or totally real if and only if $\theta=0$ or $\theta=\pi / 2$, respectively.

The action of $U(1) \times U(n)$ to $C^{n}$ is defined by

$$
(z, A) v=z v A^{*}
$$

for $(z, A) \in U(1) \times U(n)$ and $v \in C^{n}$.

Lemma 2.2. Let $G_{\theta}$ be the set of real 2-dimensional vector subspaces with Kähler angle $\theta$ of $C^{n}$. Then $U(1) \times U(n)$ acts transitively on $G_{\theta}$.

Proof. Take $V \in G_{\theta}$ and its orthonormal basis $e_{1}, e_{2}$ such that $\left\langle J e_{1}, e_{2}\right\rangle=$ $\cos \theta$. For any $(z, A) \in U(1) \times U(n), z e_{1} A^{*}, z e_{2} A^{*}$ is an orthonormal basis of $z V A^{*}$. Moreover $z V A^{*} \in G_{\theta}$, since $\left\langle J\left(z e_{1} A^{*}\right), z e_{2} A^{*}\right\rangle=\cos \theta$.

By the action of $U(n)$, we can transport $e_{1}$ to $(1,0, \ldots, 0)$. We decompose $e_{2}$ as

$$
e_{2}=\left(z_{1}, 0, \ldots, 0\right)+\left(0, z_{2}, \ldots, z_{n}\right) .
$$


Put $e_{2}^{\prime}=\left(z_{1}, 0, \ldots, 0\right)$ and $e_{2}^{\prime \prime}=\left(0, z_{2}, \ldots, z_{n}\right)$. Then we can transport $e_{2}^{\prime \prime}$ to $\left(0,\left|e_{2}^{\prime \prime}\right|, 0, \ldots, 0\right)$ by the action of $U(n-1)$. Set $e_{2}^{\prime}=(z, 0, \ldots, 0)$. Since $U(n)$ preserves the inner product and the Kähler angle, we can transport $e_{2}$ to

$$
(\sqrt{-1} \cos \theta, \sin \theta, 0, \ldots, 0)
$$

by the action of $U(n)$. Thus we can transport any $V$ in $G_{\theta}$ to $V_{\theta}$ by the action of $U(n)$, where

$$
V_{\theta}=\operatorname{Span}_{R}\{(1,0, \ldots, 0),(\sqrt{-1} \cos \theta, \sin \theta, 0, \ldots, 0)\} .
$$

Therefore $U(1) \times U(n)$ acts transitively on $G_{\theta}$.

COROllary 2.3. For a real 2-dimensional vector subspace $V$ in $\boldsymbol{C}^{n}$, $\sigma_{U(1) \times U(n)}\left(V, C^{n-1}\right)$ is dependent only on the Kähler angle of $V$.

\section{Poincaré Formulas of Real Surfaces in Complex Projective Spaces}

Let $C P^{n}$ be an $n$-dimensional complex projective space with almost complex structure $J$ and let $M$ a real 2-dimensional submanifold of $C P^{n}$. For $x$ in $M$ let $\theta_{x}$ be the Kähler angle of $T_{x} M$ in $T_{x} C P^{n}$. This is a function on $M$. We call $\theta_{x}$ the Kähler angle of $M$ at $x$.

Take a complex submanifold $N$ of complex dimension $n-1$. By Theorem 1.2 , we have

$$
\begin{aligned}
\int_{U(n+1)} & \sharp(M \cap g N) d \mu_{U(n+1)}(g) \\
= & \iint_{M \times N} \sigma_{U(1) \times U(n)}\left(T_{x} M, T_{y} N\right) d \mu_{M \times N}(x, y) .
\end{aligned}
$$

We can simply write

$$
\sigma\left(\theta_{x}, n\right)=\sigma_{U(1) \times U(n)}\left(T_{x} M, T_{y} N\right)
$$

by Corollary 2.3. We shall identify the tangent space of $C P^{n}$ with $\boldsymbol{C}^{n}$ and that of $N$ with $C^{n-1}$. By the action of $U(1) \times U(n)$ we can identify $T_{x} M$ with $V_{\theta}$ defined in the proof of Lemma 2.2. Then

$$
\begin{aligned}
\sigma(\theta, n) & =\int_{U(1) \times U(n)} \sigma\left(k^{-1} V_{\theta}, C^{n-1}\right) d \mu_{U(1) \times U(n)}(k) \\
& =\operatorname{vol}(U(1)) \int_{U(n)} \sigma\left(k^{-1} V_{\theta}, C^{n-1}\right) d \mu_{U(n)}(k) .
\end{aligned}
$$


The one parameter subgroup $e^{\sqrt{-1}} t$ in $U(1)$ corresponds to $\sqrt{-1}$ in the Lie algebra $\mathfrak{u}(1)$, whose length is $1 / \sqrt{2}$ with respect to the invariant inner product. So $\operatorname{vol}(U(1))=2 \pi / \sqrt{2}$.

We put $e_{1}=(1,0, \ldots, 0)$ and $e(\theta)=(\sqrt{-1} \cos \theta, \sin \theta, 0, \ldots, 0)$. Let $e_{1}, \ldots, e_{n}$ be the standard unitary basis of $\boldsymbol{C}^{n}$. For $k=\left(k_{i j}\right) \in U(n)$ we have

$$
\begin{aligned}
\sigma\left(k^{-1}\right. & \left.V_{\theta}, C^{n-1}\right) \\
& =\left|k^{-1} e_{1} \wedge k^{-1} e(\theta) \wedge e_{2} \wedge \sqrt{-1} e_{2} \wedge \cdots \wedge e_{n} \wedge \sqrt{-1} e_{n}\right| \\
& =\left|e_{1} k \wedge e(\theta) k \wedge e_{2} \wedge \sqrt{-1} e_{2} \wedge \cdots \wedge e_{n} \wedge \sqrt{-1} e_{n}\right| \\
& =\left|k_{1} \wedge\left(\sqrt{-1} \cos \theta k_{1}+\sin \theta k_{2}\right) \wedge e_{2} \wedge \sqrt{-1} e_{2} \wedge \cdots \wedge e_{n} \wedge \sqrt{-1} e_{n}\right| \\
& =\left|k_{11} e_{1} \wedge\left(\sqrt{-1} \cos \theta k_{11} e_{1}+\sin \theta k_{21} e_{1}\right) \wedge e_{2} \wedge \cdots \wedge \sqrt{-1} e_{n}\right| \\
& =\left.|| k_{11}\right|^{2} \cos \theta-\mathscr{I}\left(k_{11} \bar{k}_{21}\right) \sin \theta \mid
\end{aligned}
$$

where $k_{1}$ and $k_{2}$ are the first two columns of $k$ and $\mathscr{I}(z)$ is imaginary part of complex number $z$. Thus we have

$$
\sigma(\theta, n)=\left.\frac{2 \pi}{\sqrt{2}} \int_{U(n)}|| k_{11}\right|^{2} \cos \theta-\mathscr{I}\left(k_{11} \bar{k}_{21}\right) \sin \theta \mid d \mu_{U(n)}(k) .
$$

The natural projection $p: U(n) \rightarrow S^{2 n-1} \subset C^{n} ; k \mapsto k^{t}(1,0, \ldots, 0)$ is $U(n)$ equivariant, so $J d p$ is constant on $U(n)$.

$$
\sqrt{2}\left[\begin{array}{cc}
\sqrt{-1} & 0 \\
0 & 0
\end{array}\right], \quad\left[\begin{array}{cc}
0 & -e_{i}^{*} \\
e_{i} & 0
\end{array}\right], \quad \sqrt{-1}\left[\begin{array}{cc}
0 & e_{i}^{*} \\
e_{i} & 0
\end{array}\right] \quad(1 \leq i \leq n-1)
$$

is an orthonormal basis of $\left(\operatorname{Ker} d p_{e}\right)^{\perp}$. Using Proposition 2.1 we get $J d p=$ $J d p_{e}=\sqrt{2}$. By the coarea formula we have

$$
\begin{aligned}
& \left.\sqrt{2} \int_{U(n)}|| k_{11}\right|^{2} \cos \theta-\mathscr{I}\left(k_{11} \bar{k}_{21}\right) \sin \theta \mid d \mu_{U(n)}(k) \\
& \quad=\left.\operatorname{vol}(U(n-1)) \int_{S^{2 n-1}}|| z_{1}\right|^{2} \cos \theta-\mathscr{I}\left(z_{1} \bar{z}_{2}\right) \sin \theta \mid d \mu_{S^{2 n-1}}\left[\begin{array}{c}
z_{1} \\
\vdots \\
z_{n}
\end{array}\right] .
\end{aligned}
$$

Define a mapping $f: S^{2 n-1}-\left\{z \mid z={ }^{t}\left(0,0, z_{3}, \ldots, z_{n}\right) \in S^{2 n-1}\right\} \rightarrow S^{3}$ by

$$
f(z)=f\left({ }^{t}\left(z_{1}, z_{2}, z_{3}, \ldots, z_{n}\right)\right)=\frac{1}{\sqrt{\left|z_{1}\right|^{2}+\left|z_{2}\right|^{2}}}\left[\begin{array}{l}
z_{1} \\
z_{2}
\end{array}\right]=w .
$$


By the coarea formula, for a function $\phi$ defined on $S^{2 n-1}$ we have

$$
\int_{S^{3}}\left(\int_{f^{-1}(w)} \phi(z) d \mu_{f^{-1}(w)}(z)\right) d \mu_{S^{3}}(w)=\int_{S^{2 n-1}} \phi(z) J d f_{z} d \mu_{S^{2 n-1}}(z) .
$$

We first compute $J d f_{z}$ at a point $z={ }^{t}\left(z_{1}, z_{2}, z_{3}, \ldots, z_{n}\right)$. Since the mapping $f$ is $O(4) \times O(2 n-4)$-equivariant, it is sufficient to compute $J d f_{x}$ at

$$
x={ }^{t}(\cos \alpha, 0,0,0, \sin \alpha, 0, \ldots, 0)
$$

where $\cos \alpha=\sqrt{\left|z_{1}\right|^{2}+\left|z_{2}\right|^{2}}$ and $\sin \alpha=\sqrt{\left|z_{3}\right|^{2}+\cdots+\left|z_{n}\right|^{2}}$. Put

$$
y={ }^{t}(-\sin \alpha, 0,0,0, \cos \alpha, 0, \ldots, 0) \text {. }
$$

Then

$$
y, e_{i}, e_{j} \quad(2 \leq i \leq 4,6 \leq j \leq 2 n)
$$

is an orthonormal basis of $T_{x} S^{2 n-1}$. It is obvious that $y, e_{j}(6 \leq j \leq 2 n)$ are elements of $\operatorname{Ker} d f_{x}$. Using Proposition 2.1, we get

$$
J d f_{z}=\frac{1}{\left(\left|z_{1}\right|^{2}+\left|z_{2}\right|^{2}\right)^{3 / 2}} .
$$

Hence we put

$$
\phi(z)=\left.\left(\left|z_{1}\right|^{2}+\left|z_{2}\right|^{2}\right)^{3 / 2} \cdot|| z_{1}\right|^{2} \cos \theta-\mathscr{I}\left(z_{1} \bar{z}_{2}\right) \sin \theta \mid,
$$

then we have the following equation.

$$
\begin{aligned}
& \int_{S^{2 n-1}}\left.|| z_{1}\right|^{2} \cos \theta-\mathscr{I}\left(z_{1} \bar{z}_{2}\right) \sin \theta \mid d \mu_{S^{2 n-1}}(z) \\
& \quad=\left.\int_{S^{3}} \int_{f^{-1}(w)}\left(\left|z_{1}\right|^{2}+\left|z_{2}\right|^{2}\right)^{3 / 2}|| z_{1}\right|^{2} \cos \theta-\mathscr{I}\left(z_{1} \bar{z}_{2}\right) \sin \theta \mid d \mu_{f^{-1}(w)}(z) d \mu_{S^{3}}(w) .
\end{aligned}
$$

We note that for any $w \in S^{3}, f^{-1}(w)$ is equal to the set

$$
\left\{{ }^{t}\left(r w_{1}, r w_{2}, \sqrt{1-r^{2}} z_{3}, \ldots, \sqrt{1-r^{2}} z_{n}\right) \mid 0<r \leq 1,{ }^{t}\left(z_{3}, \ldots, z_{n}\right) \in S^{2 n-5}\right\} .
$$

Here we define a mapping $g:(0,1] \times S^{2 n-5} \rightarrow f^{-1}(w)$ by

$$
z^{\prime}={ }^{t}\left(r,\left(z_{3}, \ldots, z_{n}\right)\right) \mapsto{ }^{t}\left(r w_{1}, r w_{2}, \sqrt{1-r^{2}} z_{3}, \ldots, \sqrt{1-r^{2}} z_{n}\right) .
$$

It is clear that $g$ is a diffeomorphism. By a simple calculation, we get

$$
J d g_{z^{\prime}}=\left(1-r^{2}\right)^{n-3} .
$$


By the coarea formula,

$$
\begin{aligned}
\int_{f^{-1}(w)} \phi(z) d \mu_{f^{-1}(w)}(z) \\
\quad=\iint_{(0,1] \times S^{2 n-5}}(\phi \circ g)\left(z^{\prime}\right) J g\left(z^{\prime}\right) d \mu_{(0,1] \times S^{2 n-5}}\left(z^{\prime}\right) \\
=\left.|| w_{1}\right|^{2} \cos \theta-\mathscr{I}\left(w_{1} \bar{w}_{2}\right) \sin \theta \mid \operatorname{vol}\left(S^{2 n-5}\right) \int_{0}^{1} r^{5} \cdot\left(1-r^{2}\right)^{n-3} d r \\
=\left.\frac{\operatorname{vol}\left(S^{2 n-5}\right)}{n(n-1)(n-2)} \cdot|| w_{1}\right|^{2} \cos \theta-\mathscr{I}\left(w_{1} \bar{w}_{2}\right) \sin \theta \mid
\end{aligned}
$$

So we obtain

$$
\begin{aligned}
\int_{S^{2 n-1}} & \left.|| z_{1}\right|^{2} \cos \theta-\mathscr{I}\left(z_{1} \bar{z}_{2}\right) \sin \theta \mid d \mu_{S^{2 n-1}}(z) \\
\quad= & \left.\frac{\operatorname{vol}\left(S^{2 n-5}\right)}{n(n-1)(n-2)} \int_{S^{3}}|| w_{1}\right|^{2} \cos \theta-\mathscr{I}\left(w_{1} \bar{w}_{2}\right) \sin \theta \mid d \mu_{S^{3}}(w)
\end{aligned}
$$

Since

$$
\left.|| \alpha z_{1}\right|^{2} \cos \theta-\mathscr{I}\left(\left(\alpha z_{1}\right) \cdot \overline{\alpha z_{2}}\right) \sin \theta|=|\left|z_{1}\right|^{2} \cos \theta-\mathscr{I}\left(z_{1} \bar{z}_{2}\right) \sin \theta \mid
$$

for any $\alpha \in C$ with $|\alpha|=1$, we have

$$
\begin{aligned}
\left.\int_{S^{3}}|| z_{1}\right|^{2} \cos \theta-\mathscr{I}\left(z_{1} \bar{z}_{2}\right) \sin \theta \mid d \mu_{S^{3}}(z) \\
\quad=\left.2 \pi \int_{C P^{1}}|| z_{1}\right|^{2} \cos \theta-\mathscr{I}\left(z_{1} \bar{z}_{2}\right) \sin \theta \mid d \mu_{C P^{1}}[z] .
\end{aligned}
$$

Let $H(2, C)$ be a vector space of 2 by 2 Hermitian matrices. Define an inner product on $H(2, C)$ by

$$
(A, B)=\frac{1}{2} \operatorname{Trace}\left(A B^{*}\right)
$$

where $B^{*}$ is the conjugate transpose of $B$. We define $S_{*}^{2}$ by

$$
S_{*}^{2}=\left\{X \in H(2, C) \mid X^{2}=X, \operatorname{Trace}(X)=1\right\} .
$$

REMARK 3.1. $S_{*}^{2}$ is a 2-dimensional Riemannian submanifold in $H(2, C)$.

LEMMA 3.2. Let $h: C P^{1} \rightarrow S_{*}^{2}$ be a mapping given by 


$$
\left[\begin{array}{l}
z_{1} \\
z_{2}
\end{array}\right] \rightarrow\left[\begin{array}{ll}
z_{1} \bar{z}_{1} & z_{1} \bar{z}_{2} \\
z_{2} \bar{z}_{1} & z_{2} \bar{z}_{2}
\end{array}\right]
$$

where ${ }^{t}\left(z_{1}, z_{2}\right) \in S^{3}$. Then $h$ is an isometry.

A direct computation shows that $S_{*}^{2}$ is a sphere with center

$$
o=\left[\begin{array}{cc}
1 / 2 & 0 \\
0 & 1 / 2
\end{array}\right]
$$

and radius $1 / 2$. So we put $S^{2}(1 / 2)=S_{*}^{2}$. Set

$$
L=\{X \in H(2, C) \mid \operatorname{Trace}(X)=0\} .
$$

Then

$$
\left[\begin{array}{cc}
1 & 0 \\
0 & -1
\end{array}\right],\left[\begin{array}{ll}
0 & 1 \\
1 & 0
\end{array}\right],\left[\begin{array}{cc}
0 & \sqrt{-1} \\
-\sqrt{-1} & 0
\end{array}\right]
$$

is an orthonormal basis of $L$. Hence any point $x$ of $S^{2}(1 / 2)$ is represented by

$$
\begin{gathered}
{\left[\begin{array}{cc}
1 / 2 & 0 \\
0 & 1 / 2
\end{array}\right]+\left[\begin{array}{cc}
1 & 0 \\
0 & -1
\end{array}\right] x_{1}+\left[\begin{array}{ll}
0 & 1 \\
1 & 0
\end{array}\right] x_{2}+\left[\begin{array}{cc}
0 & \sqrt{-1} \\
-\sqrt{-1} & 0
\end{array}\right] x_{3}} \\
=\left[\begin{array}{cc}
1 / 2+x_{1} & x_{2}+\sqrt{-1} x_{3} \\
x_{2}-\sqrt{-1} x_{3} & 1 / 2-x_{1}
\end{array}\right] .
\end{gathered}
$$

Therefore we obtain

$$
\begin{aligned}
\int_{C P^{1}} & \left.|| z_{1}\right|^{2} \cos \theta-\mathscr{I}\left(z_{1} \bar{z}_{2}\right) \sin \theta \mid d \mu_{C P^{1}}[z] \\
& =\int_{S^{2}(1 / 2)}\left|\frac{1}{2} \cos \theta+x_{1} \cos \theta-x_{3} \sin \theta\right| d \mu_{S^{2}(1 / 2)}(x)
\end{aligned}
$$

By the isometric variable transformation

$$
\left[\begin{array}{l}
u_{1} \\
u_{2} \\
u_{3}
\end{array}\right]=\left[\begin{array}{ccc}
\cos \theta & 0 & -\sin \theta \\
0 & 1 & 0 \\
\sin \theta & 0 & \cos \theta
\end{array}\right]\left[\begin{array}{l}
x_{1} \\
x_{2} \\
x_{3}
\end{array}\right]
$$

the above integral is equal to

$$
\int_{S^{2}(1 / 2)}\left|\frac{1}{2} \cos \theta+u_{1}\right| d \mu_{S^{2}(1 / 2)}(u)=\int_{S^{2}(1 / 2)}\left|\frac{1}{2} \cos \theta-u_{1}\right| d \mu_{S^{2}(1 / 2)}(u) .
$$


Using the spherical coordinates, we have the following.

$$
\begin{aligned}
\int_{S^{2}(1 / 2)} & \left|\frac{1}{2} \cos \theta-u_{1}\right| d \mu_{S^{2}(1 / 2)}(u) \\
= & \int_{0}^{2 \pi} \int_{0}^{\pi}\left|\frac{1}{2} \cos \theta-\frac{1}{2} \cos \phi_{1}\right| \cdot \frac{1}{4} \sin \phi_{1} d \phi_{1} d \phi_{2} \\
= & \frac{\pi}{4}\left(1+\cos ^{2} \theta\right) .
\end{aligned}
$$

Using $\operatorname{vol}\left(S^{2 m-1}\right)=2 \pi^{m} /(m-1)$ !, we obtain

$$
\sigma(\theta, n)=\frac{\operatorname{vol}(U(n+1))}{2 \operatorname{vol}\left(C P^{1}\right) \operatorname{vol}\left(C P^{n-1}\right)}\left(1+\cos ^{2} \theta\right) .
$$

Now (3.1) implies Theorem 1.1 for a real submanifold $M$ of real dimension 2 and a complex submanifold $N$ of complex dimension $n-1$.

In the case of a real submanifold $M$ of real dimension $2 n-2$ and a complex submanifold $N$ of complex dimension 1 we can prove Theorem 1.1 using (3.1) and the above formula of $\sigma(\theta, n)$.

Remark 3.3. By the transfer principle in integral geometry (see [1] paragraph 3.5 on pages 14-15), it is clear that Theorem 1.1 holds for all complex space forms with isotropy subgroup $U(1) \times U(n)$.

\section{References}

[1] R. Howard, The kinematic formula in Riemannian homogeneous spaces, Mem. Amer. Math. Soc., No. 509, 106, 1993.

[ 2 ] H. Tasaki, Integral geometry under cut loci in compact symmetric spaces, Nagoya Math. J., 137 (1995), 33-53.

Institute of Mathematics

University of Tsukuba

Tsukuba, Ibaraki

305-8571

Japan

kang@math.tsukuba.ac.jp

tasaki@math.tsukuba.ac.jp 